\title{
Detection of weak directional coupling: Phase-dynamics approach versus state-space approach
}

\author{
Dmitry A. Smirnov \\ Saratov Branch of Institute of RadioEngineering and Electronics of the Russian Academy of Sciences, 38 Zelyonaya Street, \\ Saratov 410019, Russia \\ Ralph G. Andrzejak \\ John von Neumann-Institute for Computing, Forschungszentrum Jülich, 52425 Jülich, Germany
}

(Received 29 September 2004; published 15 March 2005)

\begin{abstract}
We compare two conceptually different approaches to the detection of weak directional couplings between two oscillatory systems from bivariate time series. The first approach is based on the analysis of the systems' phase dynamics, whereas the other one tests for interdependencies in the reconstructed state spaces of the systems. We analyze the sensitivity of both techniques to weak couplings in numerical experiments by considering couplings between almost identical as well as between significantly different nonlinear systems. We study different degrees of phase diffusion, test the robustness of the two techniques against observational noise, and investigate the influence of the time series length. Our results show that none of the two approaches is generally superior to the other, and we conclude that it is probably the combination of both techniques that would allow the most comprehensive and reliable characterization of coupled systems.
\end{abstract}

DOI: 10.1103/PhysRevE.71.036207

PACS number(s): 05.45.Tp, 05.45.Xt

\section{INTRODUCTION}

The complex interplay of dynamical systems results in a rich variety of synchronization phenomena. While often the unidirectionality of the underlying interactions is evident, bidirectional interactions appear indispensable for the functioning of other dynamics. For a wide variety of dynamics the type and direction of driving, however, cannot be deduced a priori. In particular, this problem often arises in the analysis of interactions in climatic processes [1], electric circuits [2], neuronlike oscillators [3-5], and physiological systems, such as the human cardiorespiratory system $[6,7]$, nephrons [8], or neuronal dynamics [9-21]. Hence, a reliable detection of weak directional couplings between two separate dynamical systems $X$ and $Y$ from the analysis of pairs of signals measured from them appears as a key to an advanced understanding of many dynamics in nature.

Different approaches to this aim have been developed in the framework of linear and nonlinear time series analysis and information theory. Among the nonlinear techniques a fundamental approach was developed for oscillatory processes exhibiting a single pronounced main rhythm. In such a case one can calculate instantaneous phases and amplitudes of the oscillations from the time series using techniques such as the analytic signal approach implemented via the Hilbert transform [22]. Subsequently, the phase variables can be investigated for possible interrelations. If the phases are found to be locked, phase synchronization is established indicating a strong coupling. In this synchronous regime, however, $X$ and $Y$ have lost their separateness. In consequence, coupling direction becomes nonidentifiable. Only for weaker couplings, which result in unsynchronized motions, a reliable identification of the coupling direction can be achieved using the phase variables. For this purpose, Rosenblum and Pikovsky proposed to reconstruct model equations for the phase dynamics of the two systems in order to investigate whether the phase dynamics of one oscillator is influenced by the phase of the other [23] (cf. also Ref. [6]). In its original form the evolution map approach of Ref. [23] requires very long time series for a reliable and unbiased detection of weak directional couplings, but after introducing some correction terms and confidence intervals to the coupling estimates it was made applicable to shorter time series as well [24].

In a conceptually different approach, the dynamics are assumed to evolve in some higher dimensional state space exhibiting some deterministic and possibly chaotic motion. Here, a reconstruction of the state vectors can be obtained by means of delay coordinates. If the states of the response system $Y$ are related via some function to the states of the driver system $X$, i.e., $\mathbf{y}(\mathbf{t})=\boldsymbol{\Psi}(\mathbf{x}(\mathbf{t}))$, one speaks of generalized synchronization. From the existence of $\Psi$ it follows that close states of the drive system will typically be mapped to close states of the response system. However, in case of generalized synchronization $\Psi$ is likely to be bijective, and, therefore, also close states of the response will typically be mapped to close states of the driving system [25]. In analogy to the phase-synchronized motion, the coupling direction can only be reliably estimated for the nonsynchronous regime. Here, closeness in the response implies closeness in the driver predominantly. We address this point, which might sound contraintuitive at first sight, in more detail below, and here just note that it represents a complementary criterion for the detection of weak directional couplings and that there exists a whole family of state-space approaches that directly or indirectly exploit this criterion [9,10,13,20,26-32].

Confronted with a pair of time series measured from two unknown dynamical systems it appears difficult to decide $a$ priori whether a phase dynamics approach or a state-space approach is more appropriate for the detection of a potential directional coupling of the dynamics. It is a well-known fact that weak couplings typically affect the phases while the oscillators' amplitudes can remain essentially uncorrelated [33], and one could conjecture that phase-dynamics approaches are superior to state-space approaches for any dy- 
namics for which well-defined phases exist. However, the only way to actually test such hypotheses is to compare the performance of phase-dynamics and state-space approaches in a controlled setting using mathematical model systems. This comparison was still lacking and therefore declared as the aim of the present study. We chose the extended evolution map approach by Smirnov and Bezruchko [24] (Sec. II A 1) as a representative phase-dynamics approach, and a statistics by Arnhold et al. [10] (Sec. II A 2) as a representative state-space approach. We applied both approaches to five mathematical model systems (Sec. II B) to reveal the superiority and inferiority of the one or the other approach in different settings. Our results are presented in Sec. III followed by the discussion and conclusions in Sec. IV.

\section{METHODS AND SYSTEMS}

\section{A. Methods}

In the following we will assume that we deal with two scalar time series $\left\{x_{n}\right\}$ and $\left\{y_{n}\right\}$ measured at discrete times $t_{n}$ from the systems $X$ and $Y$, respectively, with $t_{n}=n \Delta t$, for $n$ $=1, \ldots, N$, and $\Delta t$ denoting the sampling interval.

\section{Phase-dynamics approach}

The first step for any phase-dynamics approach is the estimation of instantaneous phases $\left\{\phi_{x}\left(t_{n}\right)\right\}$ and $\left\{\phi_{y}\left(t_{n}\right)\right\}$ from the time series $\left\{x_{n}\right\}$ and $\left\{y_{n}\right\}$. For this purpose, we applied the standard analytic signal approach using the Hilbert transform. It is important to keep in mind that phases are not well defined for arbitrary signals. If, however, the dynamics exhibit oscillations with a single main rhythm, then the phases are typically well defined. For a more thorough discussion of this issue we refer to Refs. [22,34].

In order to identify the coupling direction from the phase variables of two weakly coupled oscillators Rosenblum and Pikovsky proposed to test whether the future time evolution of the phase of one oscillator is influenced by the phase of the other oscillator [23]. For this purpose, one constructs a global model map, which characterizes the dependence of phase increments over a finite time interval $\tau$ on the phases themselves, in the form

$$
\begin{aligned}
& \phi_{x}(t+\tau)-\phi_{x}(t)=F_{x}\left(\phi_{x}(t), \phi_{y}(t)\right)+\xi_{x}(t), \\
& \phi_{y}(t+\tau)-\phi_{y}(t)=F_{y}\left(\phi_{y}(t), \phi_{x}(t)\right)+\xi_{y}(t),
\end{aligned}
$$

where $\phi_{x, y}(t)$ are unwrapped phases and $\xi_{x, y}$ are zero-mean random processes. $F_{x}$ is a trigonometric polynomial of the form

$$
F_{x}\left(\phi_{x}, \phi_{y}\right)=\sum_{m, n}\left[a_{m, n} \cos \left(m \phi_{x}+n \phi_{y}\right)+b_{m, n} \sin \left(m \phi_{x}+n \phi_{y}\right)\right] .
$$

$F_{y}$ is defined analogously. These equations are the difference form of rather universal stochastic differential equations [35], which describe the evolution of coupled phase oscillators, and, despite their simplicity, reflect adequately the properties of a wide range of oscillatory processes. Therefore, they were chosen in Ref. [23] as a basic object to derive coupling characteristics. Following Refs. $[23,24]$ precisely, we used third-order polynomials for $F_{x, y}$ and set the interval $\tau$ approximately equal to one basic oscillation period for all numerical examples below (namely, $\tau=20 \Delta t$ ).

The strength of the influence of the system $Y$ on the system $X$ is determined by the steepness of the dependence of $F_{x}$ on $\phi_{y}$, i.e., by $\partial F_{x} / \partial \phi_{y}$. Therefore, one defines

$$
c_{x}=\frac{1}{2 \pi^{2}} \int_{0}^{2 \pi} \int_{0}^{2 \pi}\left(\partial F_{x} / \partial \phi_{y}\right)^{2} d \phi_{x} d \phi_{y} .
$$

It can readily be shown [24] that

$$
c_{x}^{2}=\sum_{m, n} n^{2}\left(a_{m, n}^{2}+b_{m, n}^{2}\right) .
$$

Finally, the directionality index is defined as $d=\left(c_{y}\right.$ $\left.-c_{x}\right) /\left(c_{x}+c_{y}\right)$, where $c_{y}$ is defined in complete analogy to $c_{x}$. This index is positive if $X$ predominantly drives $Y$, whereas it is negative in the opposite case. It attains values of \pm 1 for strictly unidirectional coupling.

When dealing with time series, however, one has to estimate the coefficients $a_{m, n}, b_{m, n}$, e.g., via least squares. From them one derives the estimates $\hat{c}_{x}, \hat{c}_{y}$, and $\hat{d}$. While these estimates become quite exact for very long and stationary time series (1000-5000 basic periods [23]), significantly biased estimates are obtained in the case of relatively short time series. To remove these biases, modified estimators $\gamma_{x}$ and $\gamma_{y}$ characterizing the influence of $Y$ on $X$ and vice versa, respectively, were proposed in Ref. [24]. Instead of $d$ the quantity $\delta=\gamma_{y}-\gamma_{x}$ was proposed ibidem. Under the conditions of weak coupling and weak nonlinearity these estimators are unbiased even in the case of short time series (about $50-100$ basic periods). Like $d$, the quantity $\delta$ is positive if $X$ predominantly drives $Y$, whereas it is negative in the opposite case. It differs from $d$ in that it is non-normalized, resulting in better statistical properties. Moreover, an expression for its confidence interval was derived analytically [24]. In the present study we will use the characteristic $\delta$ as a representative phase-dynamics approach to coupling direction identification.

As indicated above, a reliable detection of the coupling direction can only be achieved in the nonsynchronous regime. If the coupling is strong enough to induce synchronization, then the information about the coupling direction is lost and the indices introduced above can have arbitrary values that are not related to the coupling intensity and direction. Hence, it is important to determine the overall degree of correlation between the phases of the two dynamics. For this purpose we applied the mean phase coherence [15] (cf. also Ref. [11]):

$$
R=\sqrt{\left\langle\cos \left(\phi_{x}-\phi_{y}\right)\right\rangle^{2}+\left\langle\sin \left(\phi_{x}-\phi_{y}\right)\right\rangle^{2}},
$$

where angle brackets denote averaging over time. It is symmetric in $x$ and $y$, attains the value of $R=1$ for the case of complete phase synchronization $\left(\phi_{x}-\phi_{y}=\right.$ const $)$ and tends to zero for independent oscillators. Here we restricted ourselves 
to the simple case of $1: 1$ phase synchronization, which is appropriate for all examples analyzed in this study.

\section{State-space approach}

For the implementation of the state-space approach, it is at first necessary to reconstruct the dynamics from the two scalar time series. We used delay coordinates [36]

$$
\begin{aligned}
\mathbf{x}_{n} & =\left[x_{n}, \ldots, x_{n-(m-1) \tau_{r}}\right], \\
\mathbf{y}_{n} & =\left[y_{n}, \ldots, y_{n-(m-1) \tau_{r}}\right],
\end{aligned}
$$

with an embedding dimension $m=10$ and a delay time $\tau_{r}$ $=12$ for $n=1, \ldots, N^{*}$ with $N^{*}=N-(m-1) \tau_{r}$.

Let $r_{n, j}$ and $s_{n, j}, j=1, \ldots, q$ denote the time indices of the $q=5$ nearest Euclidean neighbors of $\mathbf{x}_{n} \in X$ and of $\mathbf{y}_{n} \in Y$, respectively. Temporally correlated neighbors are excluded by means of a Theiler correction [37]: $\left|r_{n, j}-n\right|>W$ and $\mid s_{n, j}$ $-n \mid>W$ with $W=50$. For each $\mathbf{y}_{n}$, the mean-squared Euclidean distance to its $q$ nearest neighbors is given by

$$
R_{n}^{(q)}(Y)=\frac{1}{q} \sum_{j=1}^{q}\left|\mathbf{y}_{n}-\mathbf{y}_{s_{n, j}}\right|^{2},
$$

and the $\mathrm{X}$-conditioned mean-squared Euclidean distance is defined by replacing $s_{n, j}$ with $r_{n, j}$

$$
R_{n}^{(q)}(Y \mid X)=\frac{1}{q} \sum_{j=1}^{q}\left|\mathbf{y}_{n}-\mathbf{y}_{r_{n, j}}\right|^{2} .
$$

The averaged squared distance of $\mathbf{y}_{n}$ to all remaining points in $\left\{\mathbf{y}_{n}\right\}$ is given by

$$
R_{n}(Y)=\frac{1}{N^{*}-1} \sum_{j=1, j \neq n}^{N^{*}}\left|\mathbf{y}_{n}-\mathbf{y}_{j}\right|^{2} .
$$

If the dynamics of $Y$ is independent of $X$, then there is no particular relation between $r_{n, j}$ and $s_{n, j}$, and

$$
R_{n}(Y) \approx R_{n}^{(q)}(Y \mid X) \gg R_{n}^{(q)}(Y)
$$

holds. In contrast, if closeness in $X$ implies closeness in $Y$, then it follows

$$
R_{n}(Y) \gg R_{n}^{(q)}(Y \mid X) \approx R_{n}^{(q)}(Y) .
$$
[38]

Based on these considerations Arnhold et al. [10] defined

$$
H(Y \mid X)=\frac{1}{N^{*}} \sum_{n=1}^{N^{*}} \log \frac{R_{n}(Y)}{R_{n}^{(q)}(Y \mid X)} .
$$

If relation (11) holds then it follows that $H(Y \mid X) \rightarrow 0$, whereas higher values of $H(Y \mid X)$ are obtained if relation (12) is true. The quantity $H(X \mid Y)$ is defined by exchanging $X$ and $Y$ in Eq. (13) in order to test whether closeness in $Y$ implies closeness in $X$. We here use the antisymmetrized quantity

$$
H=H(X \mid Y)-H(Y \mid X)
$$

to test for asymmetric driver-response relationships. It attains positive values for unidirectional coupling from $X$ to $Y$ in the unsynchronized regime. To briefly motivate this relation we closely follow Refs. [10,25]: In the case of unidirectional coupling $X \rightarrow Y$ we have $\mathbf{y}_{\mathbf{n}+\mathbf{1}}=\mathbf{F}\left(\mathbf{x}_{\mathbf{n}}, \mathbf{y}_{\mathbf{n}}\right)$. If there is no noise and the coupling is nonsingular $\left[\operatorname{det}\left(\partial F_{i} / \partial x_{n}\right) \neq 0\right]$, then this relation can be inverted (at least locally) and can be written as $\mathbf{x}_{\mathbf{n}}=\mathbf{G}\left(\mathbf{y}_{\mathbf{n}}, \mathbf{y}_{\mathbf{n}+1}\right)$ or, after increasing the embedding dimension of $Y$, as $\mathbf{x}_{\mathbf{n}}=\phi\left(\mathbf{y}_{\mathbf{n}}\right)$. Hence, closeness in the response implies closeness in the driver, and $H(X \mid Y)$ attains high values. The opposite relation $\mathbf{y}_{\mathbf{n}}=\boldsymbol{\Psi}\left(\mathbf{x}_{\mathbf{n}}\right)$ holds only for the synchronous motion (by definition). For the unsynchronized motion closeness in the driver does not necessarily imply closeness in the response, and $H(Y \mid X)$ attains low values. Therefore, for the unsynchronized motion with a weak unidirectional coupling from $X$ to $Y$ we obtain $H(X \mid Y)$ $>H(Y \mid X)$ and $H>0$. In the present study we used the characteristic $H$ as a representative state-space approach to coupling direction identification.

As a counterpart to the mean phase coherence we used the symmetrized quantity

$$
H_{s}=\frac{H(X \mid Y)+H(Y \mid X)}{2} .
$$

to quantify the overall strength of interdependence.

\section{B. Exemplary mathematical systems}

We analyzed five different exemplary mathematical systems to explore the strengths and weaknesses of our two techniques. Our first two examples are given by unidirectionally coupled Rössler dynamics (cf. [39-41]):

$$
\begin{gathered}
\dot{x}_{1}=-\omega_{x} x_{2}-x_{3}+\varepsilon_{x}\left(y_{1}-x_{1}\right), \\
\dot{x}_{2}=\omega_{x} x_{1}+0.15 x_{2}, \\
\dot{x}_{3}=\left(x_{1}-c_{x}\right) x_{3}+0.2, \\
\dot{y}_{1}=-\omega_{y} y_{2}-y_{3}+\varepsilon_{y}\left(x_{1}-y_{1}\right), \\
\dot{y}_{2}=\omega_{y} y_{1}+0.15 y_{2}, \\
\dot{y}_{3}=\left(y_{1}-c_{y}\right) y_{3}+0.2 .
\end{gathered}
$$

Values of the parameters $\omega_{x, y}, \varepsilon_{x, y}$, and $c_{x, y}$ are specified below. Equations (16) were integrated using the standard fourth-order Runge-Kutta routine with a step size of 0.05 and a sampling interval of $\Delta t=0.3$. Time series of the $x_{1}$ and $y_{1}$ coordinates were taken as observables. For weak coupling the phases for these variables are well defined.

For the first example we used almost identical unidirectionally coupled Rössler dynamics: $c_{x}=c_{y}=10, \varepsilon_{x}=0, \varepsilon_{y}>0$ with a frequency mismatch $\nu$ defined by $\omega_{x, y}=1 \mp \nu$. Except for a narrow range of the frequency mismatch both the driver and response dynamics are in a chaotic regime, and Zheng and $\mathrm{Hu}$ reported that qualitatively different transitions from the unsynchronized to the synchronized motion take place when the coupling $\varepsilon_{y}$ is increased [41]. For small values of the frequency mismatch $(\nu<0.028)$ phase synchronization 
comes first, followed by generalized synchronization. In an intermediate range $(0.028<\nu<0.035)$ both synchronization forms arise at the same value of the coupling. For stronger detuning $(0.035<\nu)$ generalized synchronization sets in first, while phase synchronization is observed at a stronger coupling.

For the second example we used significantly different Rössler dynamics: $c_{x}=10, c_{y}=2, \omega_{x}=1.0, \omega_{y}=0.98$. For these parameters the $X$ dynamics is chaotic, and the $Y$ dynamics is periodic. We considered both directions of unidirectional driving, $\varepsilon_{x}=0, \varepsilon_{y}>0$, as well as $\varepsilon_{x}>0, \varepsilon_{y}=0$.

As the third example, we studied nonidentical unidirectionally coupled Lorenz dynamics. We used the standard fourth-order Runge-Kutta routine with a step size of 0.005 and a sampling interval of $\Delta t=0.03$ to integrate the equations

$$
\begin{gathered}
\dot{x}_{1}=10\left(x_{2}-x_{1}\right), \\
\dot{x}_{2}=40 x_{1}-x_{2}-x_{1} x_{3}, \\
\dot{x}_{3}=x_{1} x_{2}-\frac{8}{3} x_{3}, \\
\dot{y}_{1}=10\left(y_{2}-y_{1}\right)+\varepsilon\left(x_{1}-y_{1}\right), \\
\dot{y}_{2}=35 y_{1}-y_{2}-y_{1} y_{3}, \\
\dot{y}_{3}=y_{1} y_{2}-\frac{8}{3} y_{3} .
\end{gathered}
$$

Here, depending on the observable we use, we are confronted with situations of well-defined, "almost well"defined, or ill-defined phases. The variables $x_{1,2}$ and $y_{1,2}$ do not exhibit an oscillatory behavior with a single main rhythm, and the phase is not well defined for these signals. One can still apply the analytic signal approach but ends up with some meaningless quantities. The extracted phases $\phi_{x_{1,2}}$ and $\phi_{y_{1,2}}$ do not exhibit a pronounced linear trend, show frequent jumps of $\pm 2 \pi$ (corresponding to loops of the analytic signal that do not enclose the origin of the complex plane), and certainly do not reflect a phase of the underlying dynamics. On the other hand, using $x_{1}^{2}$ and $y_{1}^{2}$ as observables one can introduce some meaningful well-defined phases that are related to the oscillations around the unstable fixed points in the two "wings" of the Lorenz attractor [42]. The variables $x_{3}$ and $y_{3}$ exhibit chaotically modulated oscillations. The extracted phases $\phi_{x_{3}}$ and $\phi_{y_{3}}$ are almost well defined in the sense that they exhibit only rare jumps of $\pm 2 \pi$.

As the fourth example we used bidirectionally coupled stochastic van der Pol oscillators with a slight mismatch of the mean frequencies $\omega_{x}$ and $\omega_{y}$

$$
\begin{gathered}
\ddot{x}=0.2\left(1-x^{2}\right) \dot{x}-\omega_{x}^{2} x+\xi_{x}+0.03(y-x), \\
\ddot{y}=0.2\left(1-y^{2}\right) \dot{y}-\omega_{y}^{2} y+\xi_{y}+\varepsilon(x-y),
\end{gathered}
$$

where $\omega_{x}=1.02, \omega_{y}=0.98$, and $\xi_{x}$ and $\xi_{y}$ are independent Gaussian white noises with correlation functions $\left\langle\xi_{x}(t) \xi_{x}\left(t^{\prime}\right)\right\rangle=\left\langle\xi_{y}(t) \xi_{y}\left(t^{\prime}\right)\right\rangle=2 D \delta\left(t-t^{\prime}\right)$. We integrated the equations using the Euler scheme with a step size of $0.01 \pi$ and a sampling interval of $0.1 \pi$ and used the variables $x$ and $y$ as observables. The predominant coupling direction depends on the value of $\varepsilon$. Different noise intensities $D$ were used to study different relative degrees of determinism versus stochasticity and, in particular, different strengths of phase diffusion. At all values of $D$, however, the calculated phases were well defined, i.e., phase jumps by $\pm 2 \pi$ were absent or very rare. This example allows us to test whether our techniques are also applicable to detect asymmetries of bidirectional coupling rather than only the direction of unidirectional coupling.

The fifth dynamics are constituted by two structurally different systems, namely, a Rössler system and a stochastic van der Pol oscillator

$$
\begin{gathered}
\dot{x}_{1}=-x_{2}-x_{3}, \\
\dot{x}_{2}=x_{1}+0.15 x_{2}+\varepsilon_{x} y, \\
\dot{x}_{3}=\left(x_{1}-10\right) x_{3}+0.2, \\
\ddot{y}=0.1\left(1-y^{2}\right) \dot{y}-\omega_{y}^{2} y+\xi_{y}+\varepsilon_{y} x_{2},
\end{gathered}
$$

where $\omega_{y}=0.98, \xi_{y}$ is Gaussian white noise with correlation function $\left\langle\xi_{y}(t) \xi_{y}\left(t^{\prime}\right)\right\rangle=2 D \delta\left(t-t^{\prime}\right), \sqrt{2 D}=0.05$. The equations were integrated using the Euler scheme with a step size of $\pi / 3000$ and a sampling interval of $0.1 \pi$. The variables $x_{1}$ and $y$ were used as observables. We considered both directions of unidirectional coupling, that is $\varepsilon_{x}>0, \varepsilon_{y}=0$ as well as $\varepsilon_{x}=0, \varepsilon_{y}>0$. We did not consider bidirectional coupling because the notion of coupling direction is ill defined for structurally different bidirectionally coupled subsystems.

Before we leave this section we shall recapitulate that we used sampling intervals equal to about 0.05 basic periods of the respective systems' oscillations. Hence, we used time series with approximately 20 data points per basic period for all dynamics. Accordingly, for the calculation of $H$ and $\delta$ we used the same parameters for all dynamics (see above). We deliberately did not optimize these parameters for the respective dynamics to simulate the absence of a priori information about the underlying dynamics and to avoid in-sample optimization. If not stated explicitly otherwise, we used $N$ $=10000$ data points (i.e., about 500 basic periods) for all dynamics.

\section{Quantification of the methods' sensitivity}

As described in Sec. II A, $\delta$ and $H$ are constructed to attain positive values for driver-response relationships with direction $X \rightarrow Y$, while zero values should be obtained for uncoupled dynamics. For an ensemble of finite realizations of uncoupled dynamics one, of course, cannot expect values of exactly zero but rather values distributed according to certain probability distributions $\rho_{0}^{H}(x)$ and $\rho_{0}^{\delta}(x)$ centered at zero. That is $\int x \rho_{0}^{H}(x) d x=\int x \rho_{0}^{\delta}(x) d x=0$. For the coupled case (say, unidirectional coupling $X \rightarrow Y$ of the strength $\varepsilon$ ), one expects probability distributions $\rho_{\varepsilon}^{H}$ and $\rho_{\varepsilon}^{\delta}$ shifted toward higher values: $\int x \rho_{\varepsilon}^{H}(x) d x>0$ and $\int x \rho_{\varepsilon}^{\delta}(x) d x>0$. In order to quantify and compare the capability of $\delta$ and $H$ to detect weak couplings, we have to determine the values of $\varepsilon$ at 

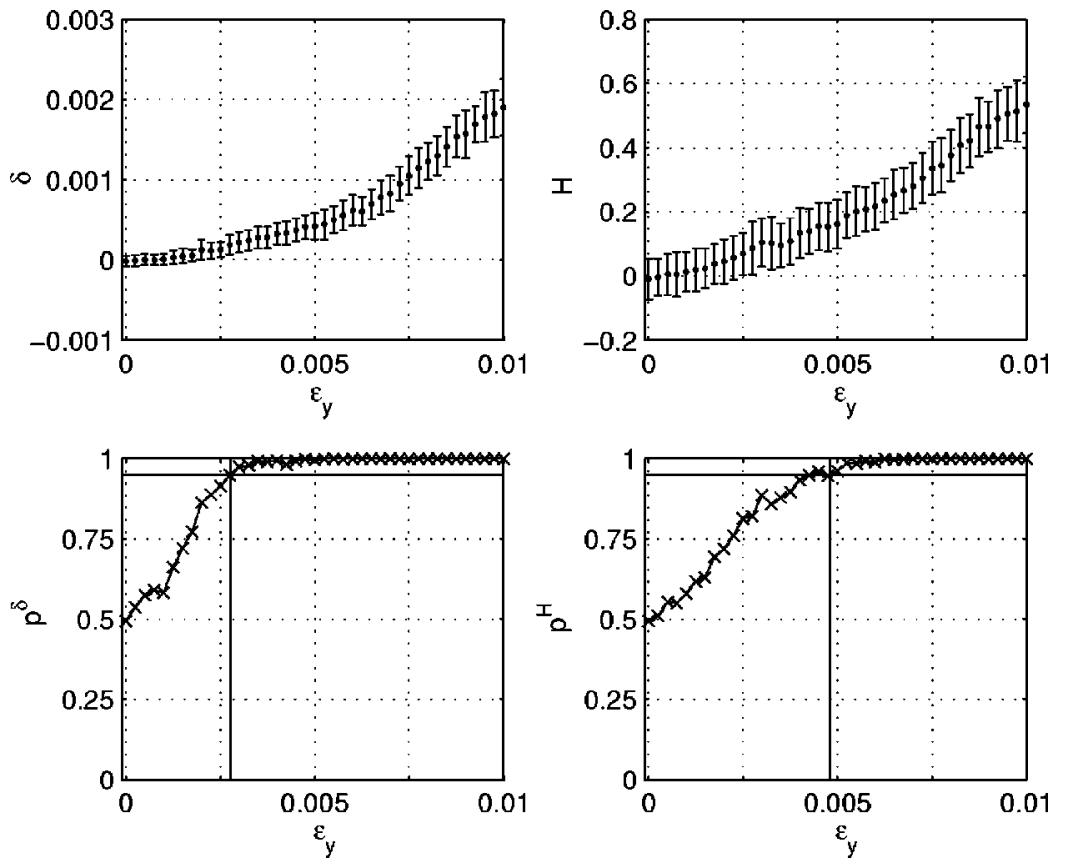

FIG. 1. Exemplary results obtained for the almost identical Rössler dynamics under variation of the coupling $\varepsilon_{y}$. Panels in the upper row show the corresponding distributions represented by their mean value plus or minus one standard deviation: upper left $\rho_{\varepsilon}^{\delta}$, upper right $\rho_{\varepsilon}^{H}$. The reference distributions $\rho_{0}^{H}$ and $\rho_{0}^{\delta}$ correspond to the leftmost distributions. Lower row: $p^{\delta}(\varepsilon)$ (left) and $p^{H}(\varepsilon)$ (right). The horizontal lines in the left and right plot represent the sensitivity thresholds $p^{\delta}(\varepsilon)=0.95$ and $p^{H}(\varepsilon)=0.95$, respectively. The vertical lines mark $\varepsilon_{95}^{\delta}$ and $\varepsilon_{95}^{H}$. In general, the curves of $p^{\delta}(\varepsilon)$ and $p^{H}(\varepsilon)$ are nonmonotonic. For the example shown here this nonmonotonicity leads to a short drop of $p^{H}(\varepsilon)$ below the sensitivity threshold after the initial crossing of this value. In these (rare) cases the second crossing was used to determine the sensitivity threshold. which $\rho_{\varepsilon}^{H}(x)$ and $\rho_{\varepsilon}^{\delta}(x)$ are significantly different from $\rho_{0}^{H}(x)$ and $\rho_{0}^{\delta}(x)$, respectively. This will be done in the following straightforward way (see also Fig. 1).

The probability that a random sample from $\rho_{\varepsilon}^{H}(x)$ has a higher value of $H$ than a random sample from $\rho_{0}^{H}(x)$ is given by

$$
p^{H}(\varepsilon)=\int_{-\infty}^{\infty}\left[\int_{-\infty}^{x^{\prime \prime}} \rho_{0}^{H}\left(x^{\prime}\right) d x^{\prime}\right] \rho_{\varepsilon}^{H}\left(x^{\prime \prime}\right) d x^{\prime \prime} .
$$

By construction we get $p^{H}(0)=0.5$, and values of $1(0)$ are obtained if every value from $\rho_{\varepsilon}^{H}(x)$ is greater (less) than every value from $\rho_{0}^{H}(x)$. These cases of nonoverlapping distributions correspond to an unambiguous discrimination of the coupled and uncoupled dynamics [43]. The increase of $p^{H}(\varepsilon)$ for low values of $\varepsilon$ reflects the sensitivity of $H$ for these weak couplings (cf. Fig. 1). The crossing of the threshold $p^{H}\left(\varepsilon_{95}\right)=0.95$ defines the value of the coupling at which we have a $5 \%$ probability to obtain a lower value for the coupled dynamics - an error probability commonly used as a threshold of significance. The analogous procedure is used for $\delta$, and the sensitivity thresholds $\varepsilon_{95}^{H}$ and $\varepsilon_{95}^{\delta}$ can be used to quantify and compare the sensitivity of the two measures for weak couplings with direction $X \rightarrow Y$ [44]. If not stated explicitly otherwise, we estimated the values of $p^{H}(\varepsilon)$ and $p^{\delta}(\varepsilon)$ using ensembles of 100 time series with initial conditions distributed according to the natural invariant measure.

\section{Estimation of phase diffusion}

A characteristic feature of oscillatory processes, which will turn out to be very important in our context, is the strength of the phase diffusion. We here briefly recall this notion and present our way of estimating a phase diffusion coefficient. Consider an ensemble of stochastic or chaotic oscillators with the same initial phase but possibly different initial amplitudes. With the evolution of the dynamics the initially identical phases will diffuse due to the effective noise caused by the stochastic or chaotic nature of the dynamics. The rate of this phase diffusion can be quantified in the following straightforward way. For the initial state the distribution of the phases has zero variance $\left[\sigma_{\phi(t=0)}=\sigma_{\phi_{0}}\right.$ $=0]$. With progressing time the variance grows, and for small times this growth can be fitted by $\sigma_{\phi}^{2}(t) \approx c t$. The quantity $2 c$ is called the phase diffusion coefficient [33].

In our context there is another quite straightforward way to estimate the phase diffusion coefficient: As a byproduct of the evolution map approach, it can readily be estimated from the residuals of the reconstructed model equations. For the uncoupled case we approximate the dependence of the phase increment $\phi(t+\tau)-\phi(t)$ on its phase $\phi(t)$ by a function $F(\phi)$. This is done by minimizing the mean-squared error $\hat{\sigma}^{2}$ $=\left\langle[\phi(t+\tau)-\phi(t)-F(\phi(t))]^{2}\right\rangle$. The minimum of $\hat{\sigma}^{2}$ is an estimate of the phase variance increment over the time interval $\tau: \hat{\sigma}_{\text {min }}^{2} \approx c \tau$. As stated before, $\tau$ is approximately one basic period. We quantified the phase diffusion directly by the value $\hat{\sigma}_{\text {min }}^{2}$, which represents the phase diffusion coefficient multiplied by one basic period and divided by 2 [45]. For all dynamics investigated here these values are listed in Table I.

\section{RESULTS}

\section{A. Unidirectionally coupled almost identical Rössler dynamics}

Figure 1 shows results obtained for the almost identical Rössler dynamics $(\nu=0.03, N=10000)$. Both $\delta$ and $H$ correctly identify the weak unidirectional coupling between the two chaotic oscillators: Both measures attain values distributed around zero for the uncoupled case and attain positive values for nonzero couplings. In consequence, both $p^{\delta}(\varepsilon)$ and $p^{H}(\varepsilon)$ grow from 0.5 to 1 . However, the increase of $p^{\delta}(\varepsilon)$ versus $\varepsilon$ is faster than the one of $p^{H}(\varepsilon)$ resulting in $\varepsilon_{95}^{\delta}$ 
TABLE I. Typical values of the phase diffusion strength $\hat{\sigma}_{\min }^{2}$ for individual uncoupled systems and relative performance of the two approaches for $N=10000$.

\begin{tabular}{lcc}
\hline \hline \multicolumn{1}{c}{ Systems } & $\hat{\sigma}_{\text {min }}^{2}$ & Superiority \\
\hline Rössler & 0.05 & $\delta$ is better \\
Lorenz & 0.8 & $H$ is better \\
van der Pol, $\sqrt{2 D}=0.01$ & 0.00008 & $\delta$ is better \\
van der Pol, $\sqrt{2 D}=0.05$ & 0.0017 & $\delta$ is better \\
van der Pol, $\sqrt{2 D}=0.1$ & 0.0068 & $\delta$ is better \\
van der Pol, $\sqrt{2 D}=0.2$ & 0.03 & $H$ is slightly better \\
van der Pol, $\sqrt{2 D}=0.4$ & 0.15 & $H$ is slightly better \\
van der Pol, $\sqrt{2 D}=0.8$ & 0.9 & $\delta$ is better \\
\hline \hline
\end{tabular}

$<\varepsilon_{95}^{H}$. In consequence, for the given time series length and frequency mismatch of the Rössler dynamics, the sensitivity of $\delta$ is higher than the one of $H$. The upper panel of Fig. 2 shows that this holds true for the whole range of frequency mismatch investigated here. Hence, for the Rössler dynamics with a well-defined phase the phase-dynamics approach $(\delta)$ appears superior to the state-space approach $(H)$.

A different picture, however, is obtained for longer time series (cf. Fig. 3). The measure $\delta$ is superior to $H$ only for short and moderate time series length, while it loses its superiority for very long time series. Hence, here we observe a superiority of $H$ despite that the phases are well defined. Furthermore, a view to the sensitivity thresholds obtained for $N=100000$ data points under variation of the frequency mismatch (Fig. 2, lower panel) shows that for this length of the time series, the measure $\delta$ is superior to $H$ for small frequency mismatches $\nu$. For an intermediate range of $\nu$ both measures have approximately the same sensitivity, and for
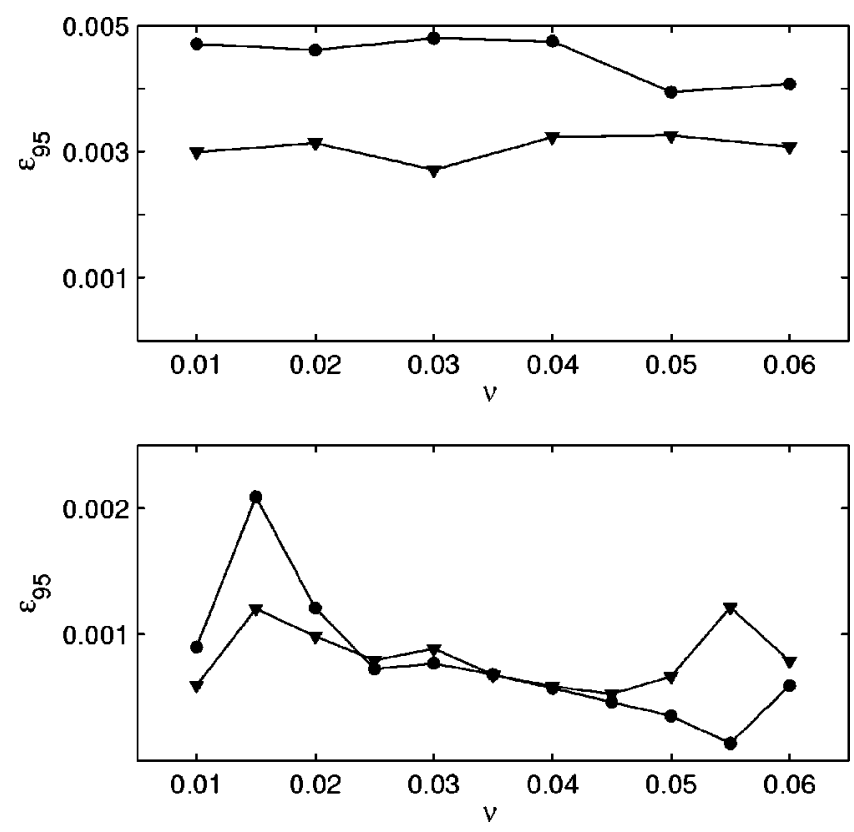

FIG. 2. Dependence of $\varepsilon_{95}^{\delta}$ (triangles) and $\varepsilon_{95}^{H}$ (circles) on the frequency mismatch $\nu$ of the Rössler dynamics for $N=10000$ (upper panel) and $N=100000$ (lower panel).

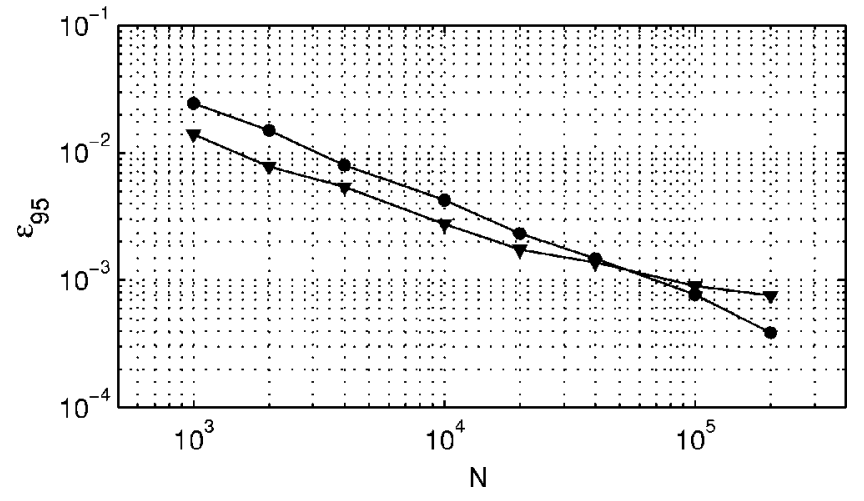

FIG. 3. Dependence of $\varepsilon_{95}^{\delta}$ (triangles) and $\varepsilon_{95}^{H}$ (circles) on the number of data points for the Rössler dynamics with a frequency mismatch of $\nu=0.03$.

high values of the frequency mismatch $H$ is superior to $\delta$. The respective ranges of the frequency mismatch approximately match those belonging to the different synchronization transitions reported in Ref. [41] and given in Sec. II B. This might suggest a relation between the kind of synchronization transition and the superiority of the one or the other method. However, it is important to recall that the curves in the upper panel of Fig. 2 do not intersect. Furthermore, if we would use some other representative phase-dynamics and/or state-space measures instead of $\delta$ and $H$, respectively, or if we would use different parameters for $\delta$ and $H$, then the curves shown in the lower panel of Fig. 2 might well be shifted and have different forms. Therefore, the relation and the relative performance of our two measures should be regarded as a very subtle factor that deserves further studies.

For the frequency mismatch of $\nu=0.015$ an outstanding high value for $\varepsilon_{95}^{H}$ is found. For an approximate range of $0.011<\nu<0.021$ the dynamics of the drive system exhibits a periodic solution (results not shown). In consequence, the response also passes through periodic windows with increasing coupling strength, starting at couplings as low as $\varepsilon$ $=0.07$ [46]. At even lower couplings the measure $H$ encounters difficulties to detect the coupling direction for this example of a chaotic Rössler dynamics driven by a periodic one.

To compare the robustness of $\delta$ and $H$ against observational noise we superimposed the driver and response dynamics of the almost identical Rössler dynamics $(\nu=0.03)$ with uncorrelated additive Gaussian noise and calculated the sensitivity thresholds of both measures for different noise levels. The latter was quantified using the ratio of the variances

$$
\xi=\frac{\sigma_{\text {noise }}^{2}}{\sigma_{\text {signal }}^{2}} .
$$

An increase in the values of $\xi$, of course, leads to increasing values of both $\varepsilon_{95}^{\delta}$ and $\varepsilon_{95}^{H}$, and only up to certain maximal noise levels is the sensitivity threshold reached at all. Only up to these $\xi_{\max }$ the respective measure allows distinguishing the coupled from the uncoupled case. From the upper panel of Fig. 4 it becomes evident that $H$ is substantially 

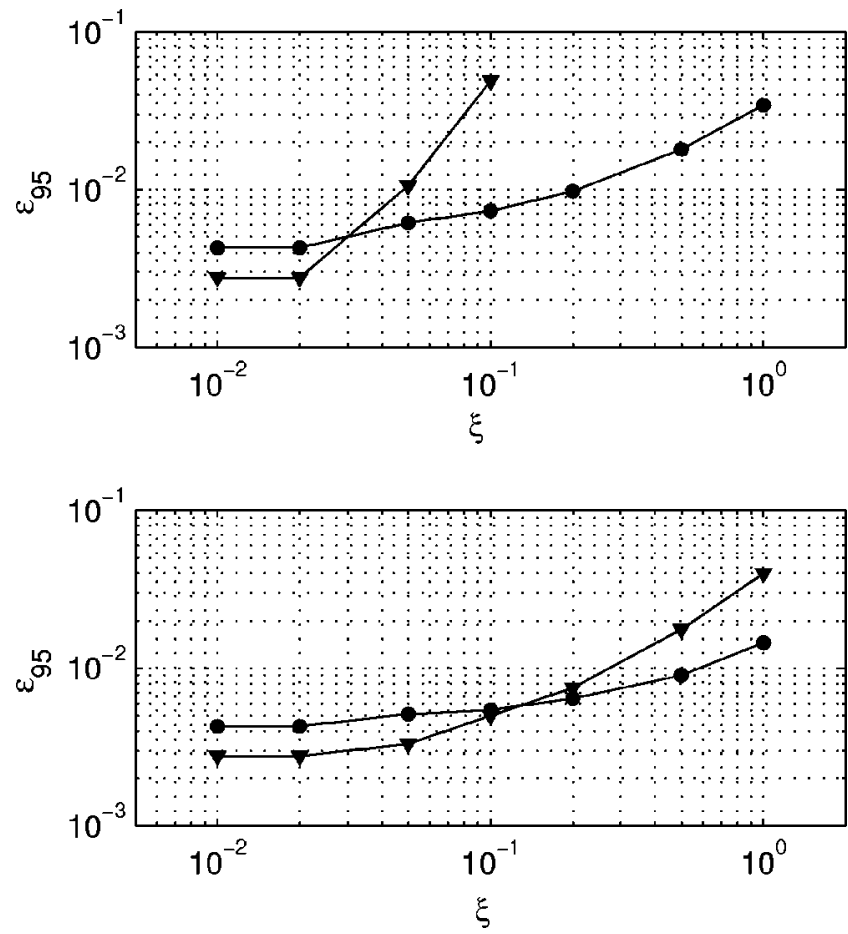

FIG. 4. Dependence of $\varepsilon_{95}^{\delta}$ (triangles) and $\varepsilon_{95}^{H}$ (circles) on the amplitude of superimposed noise for the Rössler dynamics $(\nu$ $=0.03$ ). In the upper (lower) panel results are shown for unfiltered (filtered) time series.

more robust against uncorrelated Gaussian noise. Although $H$ reaches the sensitivity threshold for values of up to $\xi_{\max }$ $=1$, the measure $\delta$ does so only up to $\xi_{\max }=0.1$. In the next step we first filtered the noisy signals using a low-pass butterworth filter with a cutoff frequency of one-fifth of the signals' bandwidth, i.e., approximately twice the mean frequency of the Rössler dynamics. The lower panel of Fig. 4 shows, according to expectation, that the sensitivity of both approaches is increased again due to the filtering, but also after the filtering $H$ is more robust against noise.

Before we proceed to results obtained for the other dynamical systems we would like to illustrate the principal limitation of the applicability of both $\delta$ and $H$ to the nonsynchronous motion. In Fig. 5 the curves of $p^{\delta}(\varepsilon)$ and $p^{H}(\varepsilon)$ for the Rössler dynamics are shown for a much wider range of the coupling strength. As indicated by the increase of the mean phase coherence and the overall nonlinear interdependence $H_{s}$, the transition to synchronization (both phase and generalized one) takes place at the coupling strength of $\varepsilon$ $\approx 0.08$. At this transition both $p^{\delta}(\varepsilon)$ and $p^{H}(\varepsilon)$ drop from values of 1 to 0 ; at even higher values of the coupling, both curves rise again. This example shows that a reliable characterization of the coupling direction cannot be obtained in the synchronous regime of the dynamics or close to it.

\section{B. Unidirectionally coupled Lorenz dynamics}

For the Lorenz dynamics the sensitivity of $H$ for weak directional couplings is higher than the one of $\delta$ regardless of what variable is used for the calculation (Fig. 6). For the observables $x_{1}, y_{1}$, the phase-dynamics approach cannot detect the coupling at all; it does not reach the sensitivity threshold within the range of the coupling investigated here. This finding is in accordance with expectation if we recall that here the phases extracted from $x_{1}$ and $y_{1}$ via the analytic signal approach are ill defined. In contrast, the state-space approach has the highest sensitivity for the observables $x_{1}, y_{1}$ $\left(\varepsilon_{95}^{H}=0.58\right)$. For the observables $x_{3}, y_{3}$, for which the phases are almost well defined, $\delta$ can detect the directional coupling but it is significantly less sensitive than $H\left(\varepsilon_{95}^{\delta}=6.50\right.$ versus $\left.\varepsilon_{95}^{H}=1.41\right)$. For $x_{1}^{2}, y_{1}^{2}$, for which the phases are well defined, the phase-dynamics approach becomes more sensitive but is still far less sensitive than the state-space approach $\left(\varepsilon_{95}^{\delta}\right.$
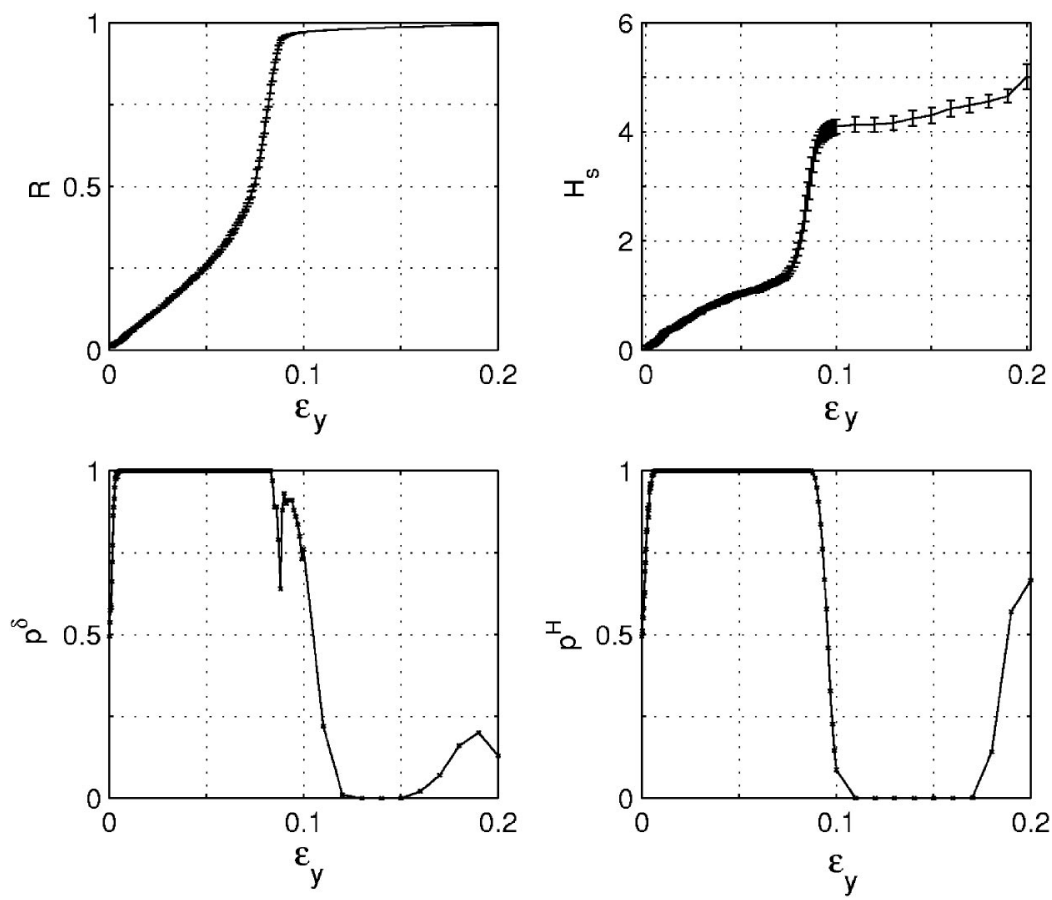

FIG. 5. Upper row: Mean phase coherence (left) and $H_{s}$ (right) versus the coupling strength $\varepsilon$ of the Rössler dynamics $(N=10000, \nu=0.03)$. Lower row: $p^{\delta}(\varepsilon)$ (left) and $p^{H}(\varepsilon)$ (right). Note that the leftmost part of the lower curves is also shown in Fig. 1. 

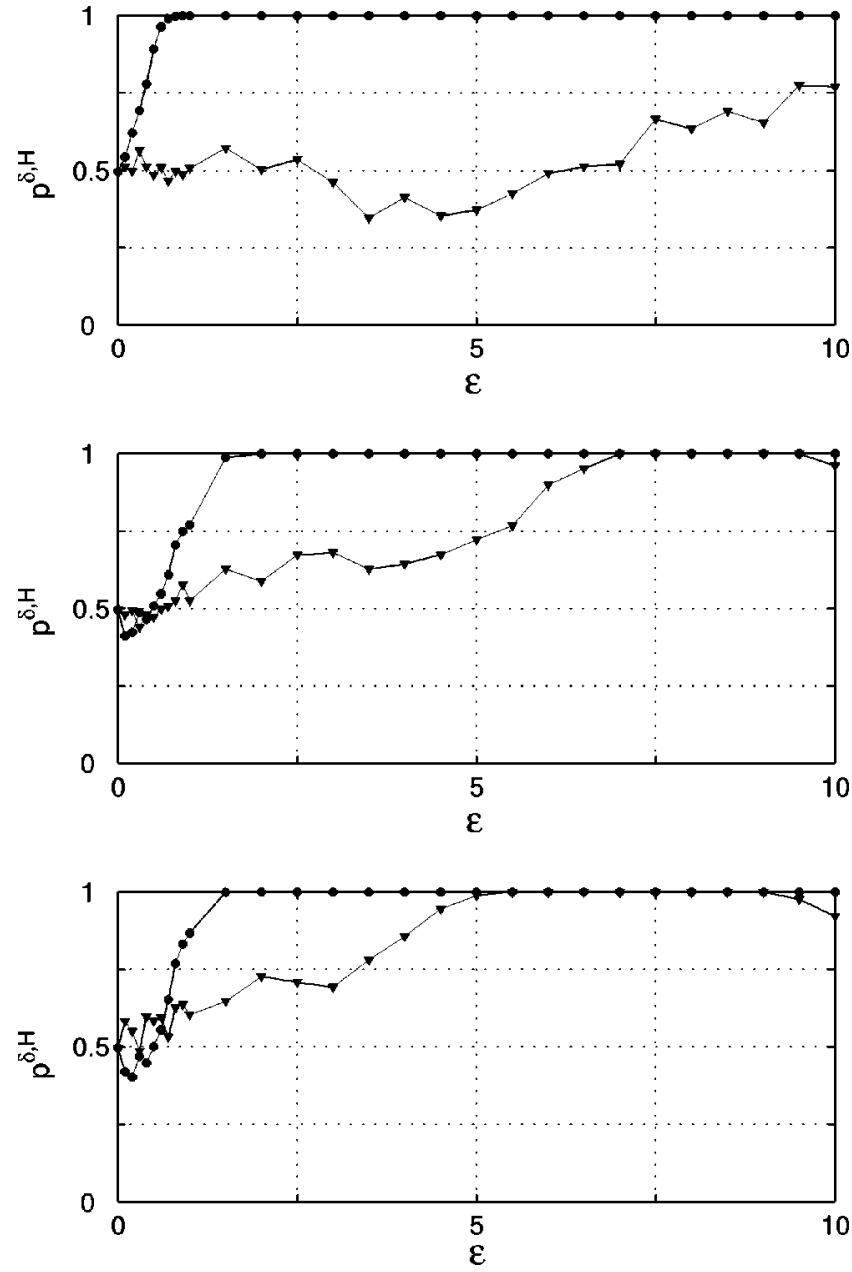

FIG. 6. Values of $p^{\delta}(\varepsilon)$ (triangles) and $p^{H}(\varepsilon)$ (circles) for the Lorenz dynamics obtained for the variables $x_{1}, y_{1}$ (top), $x_{3}, y_{3}$ (middle), and $x_{1}^{2}, y_{1}^{2}$ (bottom).
$=4.56$ versus $\left.\varepsilon_{95}^{H}=1.31\right)$. Hence, even if the phases are well defined and the time series length is moderate $(N=10000)$ a higher sensitivity of the state-space approach is observed. This finding can be explained by the very strong phase diffusion of the Lorenz dynamics (cf. Table I), which apparently strongly reduces the performance of the phase-dynamics approach.

\section{Bidirectionally coupled stochastic van der Pol oscillators}

The dependencies of $\varepsilon_{95}^{\delta, H}$ on the noise level $\sqrt{2 D}$ of the coupled van der Pol oscillators along with $p^{\delta, H}(\varepsilon)$ curves for three exemplary noise levels are depicted in (Fig. 7 [47]). Values of $\varepsilon_{95}^{\delta}$ rise monotonically with the noise level, i.e., the sensitivity decreases monotonically. As to be expected, the phase diffusion coefficients also increase with increasing noise levels (cf. Table I), again showing a strong impact of the phase diffusion on the performance of $\delta$. The dependence of $\varepsilon_{95}^{H}$ on the noise level is considerably more complicated. First of all, it exhibits a distinct minimum, the highest sensitivity is not obtained for the lowest noise level. We report here that a closer look on $H(X \mid Y)$ and $H(Y \mid X)$ reveals the reason for this finding (results not shown). For the lowest noise level investigated here $(\sqrt{2 D}=0.01)$, we, indeed, obtain the results that we expect for the unsynchronized motion: $H(X \mid Y)>H(Y \mid X) \Rightarrow H<0 \quad$ for $\quad \varepsilon<0.03$ and $H(Y \mid X)$ $>H(X \mid Y) \Rightarrow H>0$ for $\varepsilon>0.03$. However, values of both $H(X \mid Y)$ and $H(Y \mid X)$ attain almost maximal values throughout the range of the coupling $\varepsilon$ [48]. In consequence, their difference $H$ remains quite small and the sensitivity for weak directional couplings is rather low. With increasing noise the saturation of $H(Y \mid X)$ and $H(X \mid Y)$ diminishes. On the other hand, the additional noise increasingly disturbs the deterministic structure of the oscillator. While the first effect increases the sensitivity of $H$, the latter decreases it, resulting in the
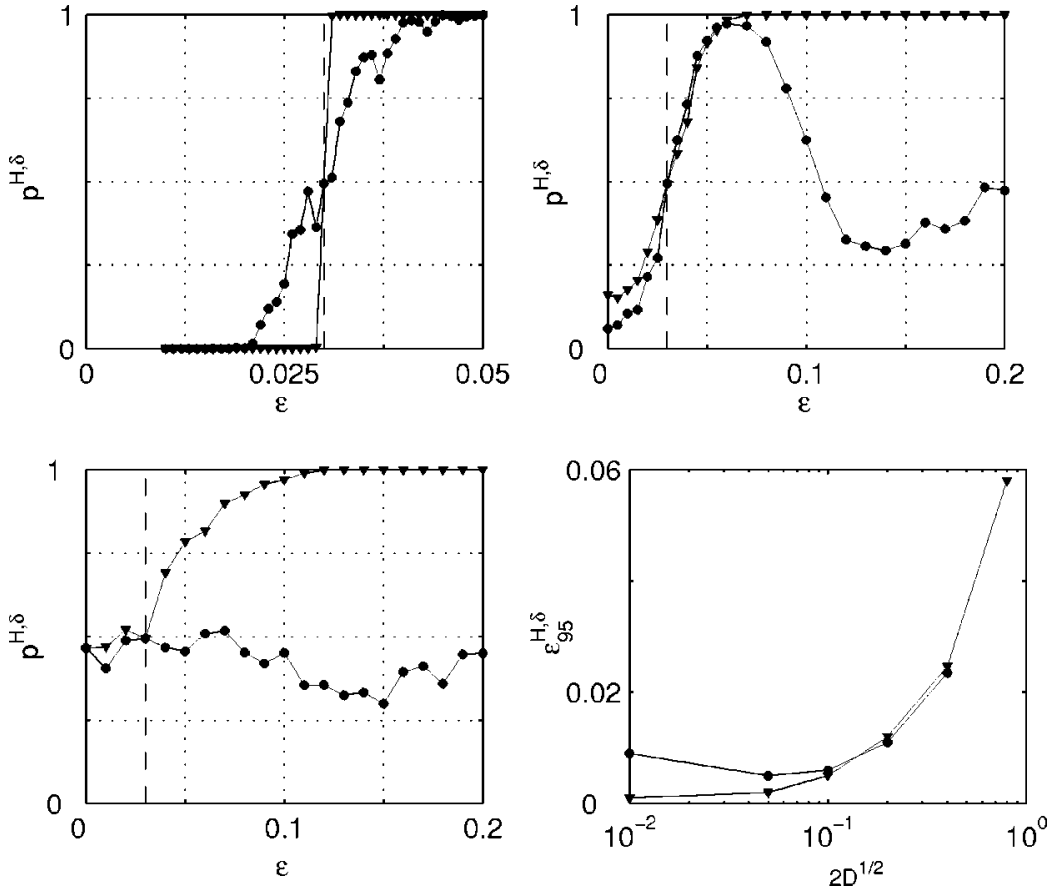

FIG. 7. Upper row and left panel of lower row: values of $\rho^{\delta}(\varepsilon)$ (triangles) and $\rho^{H}(\varepsilon)$ (circles) for the van der Pol dynamics obtained for different noise levels. From left to right and from top to bottom $\sqrt{2 D}=0.01,0.4$, and 0.8 . The vertical dashed lines mark the symmetrical coupling $(\varepsilon=0.03)$. Right panel of lower row: Dependence of sensitivity thresholds $\varepsilon_{95}^{\delta}$ (triangles) and $\varepsilon_{95}^{H}$ (circles) on the noise level. 

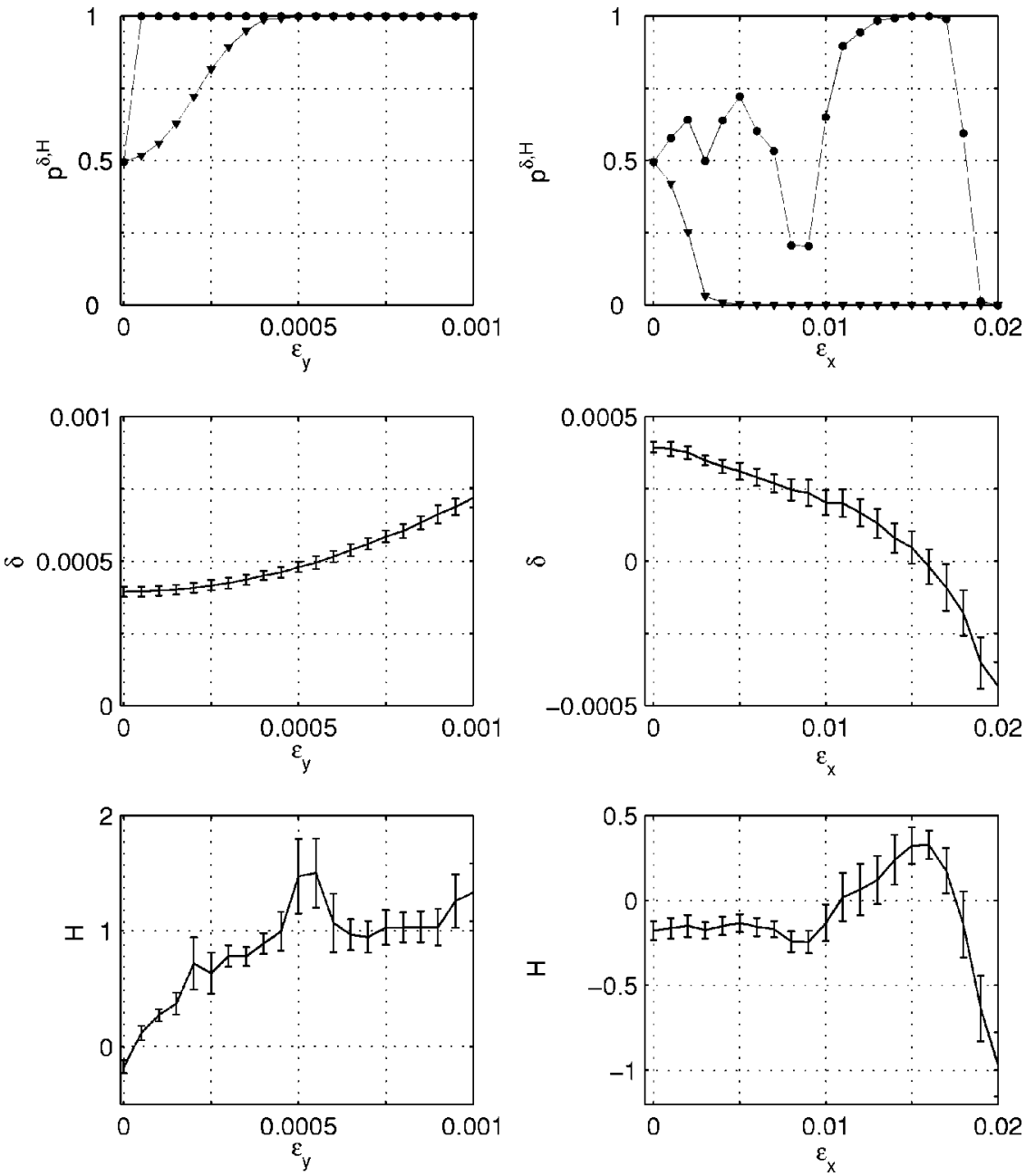

FIG. 8. Results obtained for the Rössler dynamics with significantly different parameters for both coupling directions. Upper four panels: $X \rightarrow Y$, for this coupling direction we expect positive values of $\delta$ and $H$. Correspondingly we have to use $\varepsilon_{95}$ as sensitivity threshold. Lower four panels: $Y \rightarrow X$, for this coupling direction we expect negative values of $\delta$ and $H$. Correspondingly, we have to use $\varepsilon_{05}$ as sensitivity threshold. First and third row: $\rho_{\varepsilon}^{\delta, H}$. Second and fourth row: $p^{\delta, H}(\varepsilon)$ minimum of the dependence of $\varepsilon_{95}^{\delta, H}$ on the noise level corresponding to an optimal noise level. For noise levels somewhat above this minimum the state-space approach becomes slightly superior to the phase-dynamics approach [49]. For the highest noise level investigated here $(\sqrt{2 D}=0.8)$, however, $H$ does not reach the sensitivity threshold any more, whereas $\delta$ still allows one to correctly determine the coupling direction.

\section{Couplings between significantly different dynamics}

Thus far we considered only cases of couplings between slightly different dynamics, where the notion of predominant coupling direction could readily be introduced even for bidirectional coupling. We now address the applicability of our techniques to couplings between significantly different dynamics. Here we only consider unidirectional coupling because the notion of coupling direction is ill defined for structurally different bidirectionally coupled subsystems. Results obtained for the significantly different Rössler dynamics are shown in Fig. 8. We recall that here the $X$ dynamics is chaotic, and the $Y$ dynamics is periodic. Thus, the phase diffusion coefficient is exactly zero for the $X$ dynamics (not included in Table I).

First of all, it is important to note that values of $H$ and $\delta$ obtained for the uncoupled dynamics are biased: The distri- butions $\rho_{0}^{\delta}$ and $\rho_{0}^{H}$ are not centered at zero but rather shifted toward positive and negative values, respectively. For the measure $\delta$ confidence bands were derived in Ref. [24] that readily allow one to reveal the nonsignificance of these nonzero values (results not shown). However, no such confidence bands exist for $H$, and here only the use of bivariate surrogate time series [50-52] could help to rule out spurious conclusions about the presence and/or direction of a coupling. In any case, due to the shifted reference distributions the judgement of the measures' sensitivity becomes rather problematic. If we proceed, nonetheless, we can see that for the driving direction $X \rightarrow Y$ the measure $H$ attains positive values and formally has a very high sensitivity that even surpasses the one of $\delta$. For the opposite driving direction, however, the curve $p^{H}(\varepsilon)$ first crosses the value of 0.95 , falsely indicating a coupling from $X \rightarrow Y$. This is in some analogy to results obtained for the periodic regime of the driver of the almost identical Rössler dynamics; again $H$ encounters difficulties for a chaotic Rössler dynamics driven by a periodic one. Regarding only the curves of $p_{0}^{\delta}$ suggests that the measure $\delta$ correctly detects both driving directions. However, looking at $\rho_{0}^{\delta}$ for the $Y \rightarrow X$ we see that positive values of $\delta$ are obtained up to a quite high value of $\varepsilon \approx 0.015$. We note that also this bias turns out to be insignificant with regard to the confidence bands of $\delta$. 

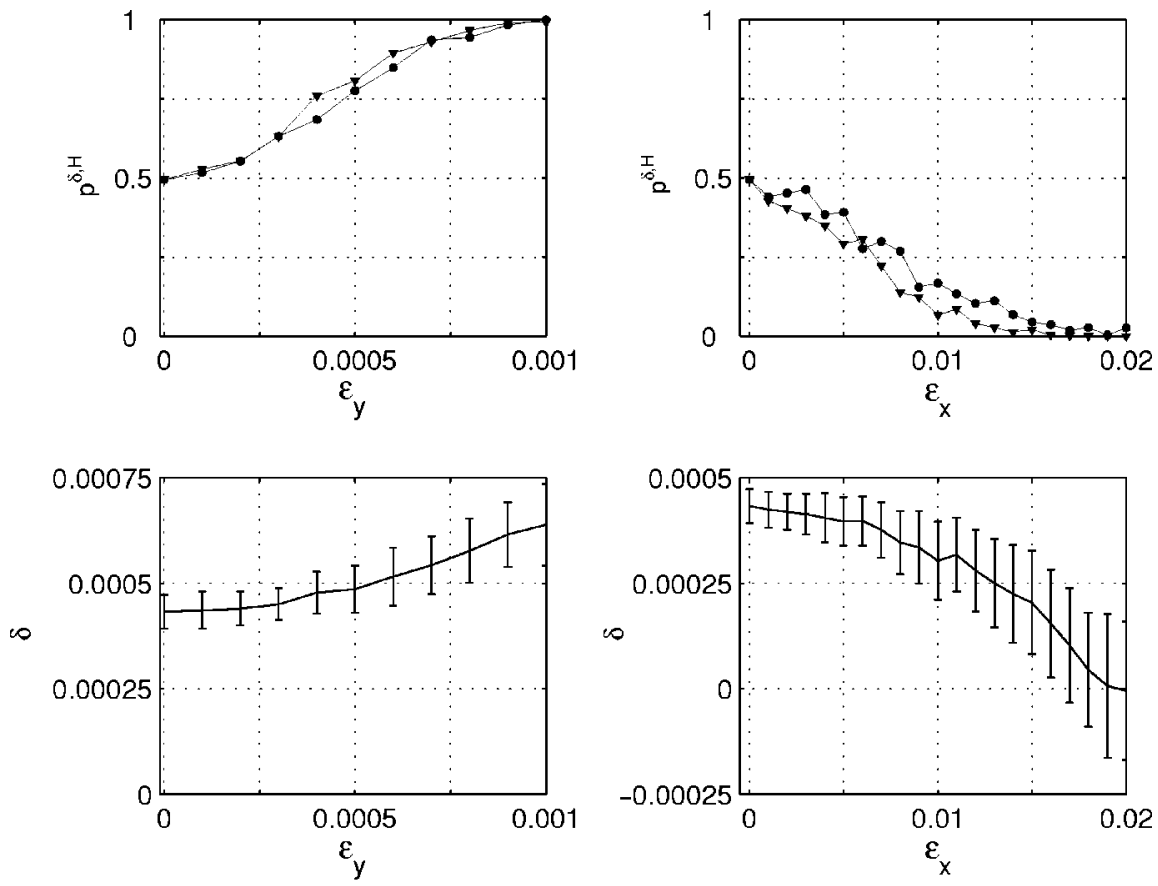

FIG. 9. Analogous to Fig. 8 but for the unidirectionally coupled Rössler-van der Pol dynamics. Upper four panels van der Pol oscillator is driving the Rössler dynamics $\left(\varepsilon_{y}=0, Y \rightarrow X\right)$. Lower four panels: Rössler dynamics is driving the van der Pol oscillator $\left(\varepsilon_{y}=0, X \rightarrow Y\right)$.

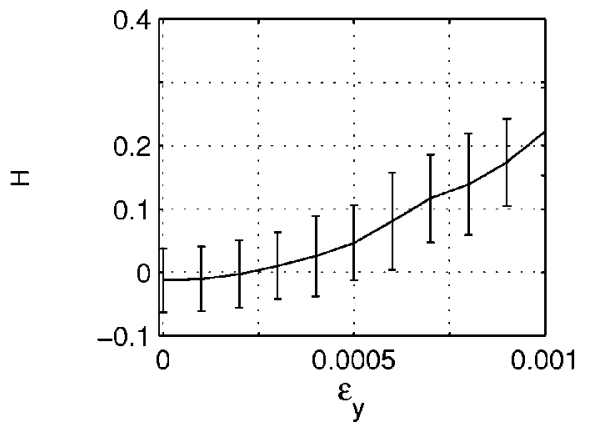

In summary, these results reveal some caveats in the application of both measures to couplings between significantly different dynamics. Therefore, in applications to real-world dynamics, which are often significantly different, one has to be very careful with the conclusion of a predominant driving direction derived from absolute values of $\delta$ and $H$. Nonetheless, relative changes of these measures could be used to trace the change of the dynamics' coupling characteristics in the course of time or under the change of some parameter of the dynamics.

The results obtained for the van der Pol-Rössler dynamics, two structurally different dynamics, are shown in Fig. 9. Looking at the curves of $p_{0}^{\delta, H}$, we see that both measures correctly detect both driving directions and that a little higher sensitivity is obtained for $\delta$. This finding is in accordance with results for the other systems with low phase diffusion. However, we should note again that in this case the values of $\delta$ obtained for the uncoupled dynamics are positively biased, whereas values of $H$ are distributed around zero. Again the confidence bands of $\delta$ reveal the nonsignificance of this positive bias (results not shown).

\section{DISCUSSION}

We presented a detailed comparison of two approaches to the detection of weak directional couplings, a representative state-space approach (characteristic $H$ ) and a representative phase-dynamics approach (characteristic $\delta$ ). We analyzed several exemplary mathematical model systems and also analyzed the influence of observational noise and the time series length on the performance of the two approaches.

First of all, we would like to recapitulate that a welldefined phase is a prerequisite for the application of any phase-dynamics approach. If no meaningful phase can be extracted, then one should only apply a state-space approach. We used different observables from the Lorenz dynamics to illustrate this evident fact. Our results, however, indicate also that a well-defined phase does not necessarily result in the superiority of the phase-dynamics approach. In contrast, we provided various examples for which we obtained superiority of the state-space approach for dynamics with well-defined phases.

As a very crucial influencing factor, we could single out the strength of the phase diffusion. The comparison of the Rössler dynamics and the different observables of the Lorenz dynamics, as well as the variation of the noise level in the van der Pol dynamics clearly revealed the lower the phase diffusion, the more sensitive is the phase-dynamics approach. For the state-space approach, the situation is a bit more complicated and one has to distinguish between phase diffusion caused by deterministic chaotic and stochastic dy- 
namics. The former does not reduce the sensitivity of the state-space approach (see again the almost identical Rössler and Lorenz dynamics). Stochasticity, however, generally lowers the sensitivity of $H$ to detect interrelations between the two dynamics (see again coupled van der Pol dynamics and the coupled van der Pol-Rössler dynamics). On the other hand, we observed an intermediate level of dynamical noise in the coupled van der Pol dynamics for which the statespace approach was approximately as sensitive as the phasedynamics approach or even slightly superior to it despite a comparably low phase diffusion. The superiority of one or the other approach probably depends on how the noise is "distributed" among the dynamics of phases and amplitudes.

The second factor that is very important is observational noise. By considering noisy time series from the coupled Rössler systems, we found the state-space approach to be considerably more robust against observational noise than the phase-dynamics approach. At the first glance, this can be regarded as a surprising result because one could expect that the extraction of the phase acts as an effective filtering. However, with an increasing level of observational noise, spurious jumps of the calculated phase by $\pm 2 \pi$ become more frequent, significantly affecting the performance of the phase-dynamics approach [53].

On the same example of the almost identical coupled Rössler dynamics, we studied the influence of the time series length, which turned out to be the third very important factor influencing the performance of the two techniques. One has to recall that the measure $H$ is calculated with a fixed number of nearest neighbors. Under an increase in the time series length, these neighbors will, on average, be found in shorter distances to the reference points. Closer neighbors are better suited to characterize the dynamics in the vicinity of the reference point, resulting in a higher sensitivity of $H$. This distance scales as $N^{-D}$, where $D$ is the fractal dimension of the attractor. As for the phase-dynamics approach, the standard deviation of the estimate $\delta$ scales as $N^{-1}$ [24], and it can be shown that the sensitivity threshold for large $N$ must scale approximately according to the same law. The first effect appears more dominant as we found the sensitivity of $H$ to increase more significantly with the time series length than the one of $\delta$, and we can conjecture that this effect will be even more pronounced for higher dimensional dynamics [54].

Moreover, in case of very long time series one can observe some more subtle factors influencing the performance of the techniques. Our results obtained for the almost identical Rössler dynamics suggest that, depending on the form of the transition to the synchronous motion, qualitatively different precursors are present in the properties of the dynamics of the driven system at very weak couplings. These different precursors can be detected best using a phase-dynamics approach (if phase synchronization comes first with an increase in coupling strength) or a state-space approach (otherwise). Certainly, the mechanism underlying the different transitions to synchronization are not completely understood, and we shall emphasize the limited conclusiveness of our results for this context. Doubtless, this interplay of generalized synchronization and phase synchronization and the different precursors found in the dynamics appears very intriguing and deserve further studies.
Let us finally note that there are some differences between the two representative techniques considered. The phasedynamics approach used here is based on a parametric representation of the systems under study, whereas the statespace approach is nonparametric. This asymmetry was not introduced by us artificially but corresponds well to the underlying ideas of both approaches. The parametric representation of the phase dynamics (trigonometric polynomials) is quite universal and flexible because of the physical constraint of the phase variable; the dependence on the phase must be $2 \pi$ periodic. Therefore, the choice of trigonometric polynomials is very natural. One could use a nonparametric approach to model the phase dynamics as well, e.g., local linear models [6], but it can be argued that such an approach would give approximately the same results and would be more vulnerable to noise and dependent on the time series length. As for the state-space approach, one could not readily use a parametric approach instead of $H$ because there is no general way to parametrize arbitrary multivariate dependencies. Furthermore, it should be noted that the measure $\delta$ tests for the influence of present phase values of one dynamics on future phase values of the other dynamics. In contrast, the measure $H$ only evaluates present states of simultaneous space vectors. Therefore, at first sight it appears more logical to compare $\delta$ to a mutual prediction error scheme [9] that also incorporates the future evolution of state-space trajectories. However, in delay coordinates the information about future state-space vectors is incorporated by simply choosing a long delay window. The information given by a present state-space vector and its future states is basically the same as the present state-space vector with a longer delay window. Hence, the information about future values is implicitly used for $H$, and, with the parameters specified above, we expect it to give basically the same results as a mutual prediction scheme. Furthermore, the measure $H$ appears conceptually more straightforward as has less parameters than a mutual prediction scheme, and no specific predictive model (e.g., locally constant versus locally linear) has to be chosen.

For the three main factors isolated here one can conclude that the phase-dynamics approach appears superior generally if the phase diffusion is weak, observational noise level is low, and the time series are not very long. Otherwise, the state-space approach appears superior. However, the relative performance of the approaches is influenced also by some more subtle factors such as stochastic or deterministic nature of the phase diffusion and the kind of transition to synchronization regimes with increase in coupling strength. Thus, neither of the two approaches investigated here is generally superior to the other, but rather both have their specific strengths and weaknesses. In applications to experimental time series one should, therefore, not favor a priori one or the other approach but rather consider applying both techniques. It is probably the combination of information from both techniques that allows the most comprehensive and reliable characterization of unknown dynamics.

An investigation of real-world dynamics, such as neuronal dynamics or genetic microarray-produced time series, shall be the subject of future investigations. In contrast to the models investigated here, neuronal dynamics do not represent pairs of coupled but nonetheless distinct and self- 
sustained oscillators. Rather, time series measured from the brain, such as the electroencephalogram, always reflect the interaction of millions of individual units organized as quasicontinuous networks. Due to the superposition of an uncounted number of degrees of freedom, even the reconstruction of the dynamics using delay coordinates and the estimation of the phase using the Hilbert transform can be problematic. For this reason it is sometimes argued that measures such as $\delta$ or $H$ should not be applied to neuronal dynamics because the underlying assumptions are violated. However, the complementary point of view, which we share, is that, in particular, these complicated properties make neuronal dynamics a very interesting field of application, and recent studies show that such applications can indeed contribute to our understanding of the brain (see, for example,
$[21,55]$ and references therein). Finally, a comparison against different information-theoretic approaches based on reconstructed state spaces [56] and/or phases [7] should also be the subject of future investigations.

\section{ACKNOWLEDGMENTS}

The authors are grateful to B. Bezruchko, P. Grassberger, A. Kraskov, T. Kreuz, and S. Astakhov for fruitful discussions of this work. D.A.S acknowledges financial support from the President of Russia (Grant No. MK-1067.2004.2), Russian Science Support Foundation, program BRHE (REC006), and RFBR (Grant No. 05-02-16305), and the kind hospitality of the John von Neumann-Institute for Computing at the Research Center Juelich.
[1] S. Jevrejeva, J. Moore, and A. Grinsted, J. Geophys. Res. 108, 4677 (2003).

[2] B. Bezruchko, V. Ponomarenko, M. G. Rosenblum, and A. S. Pikovsky, Chaos 13, 179 (2003).

[3] V. B. Kazantsev, Phys. Rev. E 64, 056210 (2001).

[4] V. B. Kazantsev, V. I. Nekorkin, V. I. Makarenko, and R. Llinas, Proc. Natl. Acad. Sci. U.S.A. 100, 13064 (2003).

[5] V. P. Zhigulin, M. I. Rabinovich, R. Huerta, and H. D. I. Abarbanel, Phys. Rev. E 67, 021901 (2003).

[6] M. G. Rosenblum, L. Cimponeriu, A. Bezerianos, A. Patzak, and R. Mrowka, Phys. Rev. E 65, 041909 (2002).

[7] M. Paluš and A. Stefanovska, Phys. Rev. E 67, 055201(R) (2003).

[8] O. V. Sosnovtseva, A. N. Pavlov, E. Mosekilde, and N. H. Holstein-Rathlou, Phys. Rev. E 66, 061909 (2002).

[9] S. J. Schiff, P. So, T. Chang, R. E. Burke, and T. Sauer, Phys. Rev. E 54, 6708 (1996).

[10] J. Arnhold, K. Lehnertz, P. Grassberger, and C. E. Elger, Physica D 134, 419 (1999).

[11] J. P. Lachaux, E. Rodriguez, J. Martinerie, and F. J. Varela, Hum. Brain Mapp 8, 194 (1999).

[12] P. Mann-Metzer and Y. Yarom, J. Neurosci. 19, 3298 (1999).

[13] M. Le Van Quyen, J. Martinerie, C. Adam, and F. Varela, Physica D 127, 250 (1999).

[14] N. Brenner, S. Strong, R. Koberle, W. Bialek, and R. R. D. van Steveninck, Neural Comput. 12, 1531 (2000).

[15] F. Mormann, K. Lehnertz, P. David, and C. E. Elger, Physica D 144, 358 (2000).

[16] H. K. M. Meeren, J. P. M. Pijn, E. L. J. M. van Luijtelaar, A. L. M. Coenen, and F. H. Lopes da Silva, J. Neurosci. 22, 1480 (2002).

[17] M. Breakspear and J. R. Terry, Neuroimage 16, 822 (2002).

[18] P. A. Tass, T. Fieseler, J. Dammers, K. Dolan, P. Morosan, M. Majtanik, F. Boers, A. Muren, K. Zilles, and G. R. Fink, Phys. Rev. Lett. 90, 088101 (2003).

[19] L. Cimponeriu, M. Rosenblum, T. Fieseler, J. Dammers, M. Schiek, M. Majtanik, P. Morosan, A. Bezerianos, and P. Tass, Prog. Theor. Phys. 150, 22 (2003).

[20] U. Feldmann and J. Bhattacharya, Int. J. Bifurcation Chaos Appl. Sci. Eng. 14, 504 (2004).
[21] F. Mormann, T. Kreuz, C. Rieke, R. G. Andrzejak, A. Kraskov, P. David, C. E. Elger, and K. Lehnertz, Clin. Neurophysiol. 116, 569 (2005).

[22] M. Rosenblum, A. Pikovsky, J. Kurths, C. Schäfer, and P. A. Tass, in Phase Synchronization: From Theory to Data Analysis, edited by F. Moss and S. Gielen (Elsevier Science, New York, 2001), pp. 279-321.

[23] M. G. Rosenblum and A. S. Pikovsky, Phys. Rev. E 64, 045202(R) (2001).

[24] D. A. Smirnov and B. P. Bezruchko, Phys. Rev. E 68, 046209 (2003).

[25] R. Quian Quiroga, J. Arnhold, and P. Grassberger, Phys. Rev. E 61, 5142 (2000).

[26] A. Cenys, G. Lasiene, and K. Pyragas, Physica D 52, 332 (1991).

[27] N. F. Rulkov, M. M. Sushchik, L. S. Tsimring, and H. D. I. Abarbanel, Phys. Rev. E 51, 980 (1995).

[28] L. Kocarev and U. Parlitz, Phys. Rev. Lett. 76, 1816 (1996).

[29] R. Quian Quiroga, A. Kraskov, T. Kreuz, and P. Grassberger, Phys. Rev. E 65, 041903 (2002).

[30] C. J. Stam and B. W. van Dijk, Physica D 163, 236 (2002).

[31] J. R. Terry and M. Breakspear, Biol. Cybern. 88, 129 (2003).

[32] J. Bhattacharya, E. Pereda, and H. Petsche, IEEE Trans. Syst. Man Cybern. 33 (Part B), 85 (2003).

[33] A. Pikovsky, M. Rosenblum, and J. Kurths, Synchronization: A Universal Concept in Nonlinear Sciences (Cambridge University Press, Cambridge, UK, 2001).

[34] V. Anishchenko, T. Vadivasova, and G. Strelkova, Fluct. Noise Lett. 4, 219 (2004).

[35] A. Pikovsky, M. Rosenblum, and J. Kurths, Int. J. Bifurcation Chaos Appl. Sci. Eng. 10, 2291 (2000).

[36] F. Takens, in Dynamical Systems and Turbulence, edited by D. A. Rand and L.-S. Young, Lecture Notes in Mathematics Vol. 898, (Springer-Verlag, Berlin, 1980), pp. 366-381.

[37] J. Theiler, Phys. Rev. A 34, 2427 (1986).

[38] In Ref. [10] an alternative measure has been proposed: $S(Y \mid X)=1 / N^{*} \sum_{n=1}^{N^{*}}\left[R_{n}^{(q)}(Y) / R_{n}^{(q)}(Y \mid X)\right]$. However, because the measure $H$ was found to be more robust against noise and easier to interpret than $S$ (cf. Ref. [25]) we restrict ourselves to the use of $H$. 
[39] O. E. Rössler, Phys. Lett. 57A, 397 (1976).

[40] M. G. Rosenblum, A. S. Pikovsky, and J. Kurths, Phys. Rev. Lett. 76, 1804 (1996).

[41] Z. Zheng and G. Hu, Phys. Rev. E 62, 7882 (2000)

[42] Note that the quantity $x_{1}^{2}$ is similar to the quantity $\sqrt{x_{1}^{2}+x_{2}^{2}}$ used along with the $x_{3}$ variable to project the Lorenz attractor to a plane in order to extract a phase as a rotation angle in Ref. [33].

[43] Note that this technique is very similar to the receiver operator characteristic [57].

[44] For the case of the opposite coupling direction $Y \rightarrow X$, one should analogously define the sensitivity threshold as $\varepsilon_{05}^{H}$ and $\varepsilon_{05}^{\delta}$ because an increase in coupling strength leads to negative values of $\delta$ and $H$ here.

[45] If the phase nonlinearity of the dynamics is very complicated, then the third-order polynomial can be too simple to describe the nonlinearity and a large mean-squared error can be obtained for dynamics with a low phase diffusion. Hence, $\hat{\sigma}_{\text {min }}^{2}$ reflects both phase diffusion and the discrepancy between the true phase nonlinearity of the oscillator and the flexibility of the model used. However, in the examples considered in our study, the phase nonlinearity is not expected to be very complicated, so $\hat{\sigma}_{\min }^{2}$ can be regarded as a good estimate of the true effective phase diffusion.

[46] This situation was not described in Ref. [41] despite this range of parameters was investigated there.

[47] Here, symmetric coupling $(\varepsilon=0.03)$ corresponds to the uncoupled dynamics of the other examples. In consequence, the distributions are compared to the basic distribution $\rho_{0.03}(x)$ rather than to $\rho_{0}(x)$. We considered values of $\varepsilon>0.03$ corresponding to the coupling direction from $X$ to $Y$ and accordingly used $\varepsilon_{95}$ as the sensitivity threshold. The opposite cou- pling direction $(\varepsilon<0.03)$ can be analyzed analogously using $\varepsilon_{05}^{H, \delta}$ as sensitivity thresholds.

[48] Recall that the measure $H$ is not normalized. Nonetheless, for a certain attractor geometry there is a maximal value $H(X \mid Y)$ that would be obtained for identical synchronization. This value is simply given by the mean ratio of the mean distances to $q$ nearest neighbors and the mean distances to all remaining points. This directly follows from Eq. (13) with $R_{n}^{(q)}(X \mid Y)$ $=R_{n}^{(q)}(Y)$ and in analogy for $H(Y \mid X)$.

[49] The drop of $p^{H}(\varepsilon)$ below the sensitivity threshold after the initial upward crossing is related to high values of the overall interdependence $H_{a}$ and mean phase coherence $R$ for this range of the coupling (results not shown).

[50] J. Theiler, S. Eubank, A. Longtin, B. Galdrikian, and J. D. Farmer, Physica D 58, 77 (1992).

[51] T. Schreiber and A. Schmitz, Physica D 142, 346 (2000).

[52] R. G. Andrzejak, A. Kraskov, H. Stögbauer, F. Mormann, and T. Kreuz, Phys. Rev. E 68, 066202 (2003).

[53] Formally, spurious phase jumps lead to high values of the estimated phase diffusion coefficient, and we again obtain a poor performance of the phase-dynamics approach for strong phase diffusion. However, the phase diffusion coefficient is overestimated here and it reflects only errors in the determination of the phase rather than a property of the oscillator's phase dynamics.

[54] This is the case for deterministic dynamics, while for the stochastic dynamics an increase in time series length does not change superiority of the techniques so drastically.

[55] M. Breakspear, Neuroinformatics 2, 205 (2004).

[56] T. Schreiber, Phys. Rev. Lett. 85, 461 (2000).

[57] J. Hanley and B. J. McNeil, Radiology 143, 29 (1982). 